\title{
PAIEMENTS POUR SERVICES ENVIRONNEMENTAUX ET BIODIVERSITÉ DANS LES PAYS DU SUD
}

Le salut par la «déforestation évitée »

Alain Karsenty, Thomas Sembres, Mino Randrianarison

Armand Colin | « Revue Tiers Monde »

2010/2 n 202 | pages 57 à 74

ISSN 1293-8882

ISBN 9782200926618

Article disponible en ligne à l'adresse :

http://www.cairn.info/revue-tiers-monde-2010-2-page-57.htm

\section{!Pour citer cet article :}

Alain Karsenty et al., « Paiements pour services environnementaux et biodiversité dans les pays du sud. Le salut par la « déforestation évitée » », Revue Tiers Monde 2010/2 (n²02), p. 57-74. DOI 10.3917/rtm.202.0057

Distribution électronique Cairn.info pour Armand Colin.

(C) Armand Colin. Tous droits réservés pour tous pays.

La reproduction ou représentation de cet article, notamment par photocopie, n'est autorisée que dans les limites des conditions générales d'utilisation du site ou, le cas échéant, des conditions générales de la licence souscrite par votre établissement. Toute autre reproduction ou représentation, en tout ou partie, sous quelque forme et de quelque manière que ce soit, est interdite sauf accord préalable et écrit de l'éditeur, en dehors des cas prévus par la législation en vigueur en France. Il est précisé que son stockage dans une base de données est également interdit. 


\title{
BIODIVERSITÉ : ENJEUX NORD-SUD
}

\section{PAIEMENTS POUR SERVICES ENVIRONNEMENTAUX ET BIODIVERSITÉ DANS LES PAYS DU SUD}

Le salut par la « déforestation évitée »?

\author{
Alain Karsenty ${ }^{\star}$, Thomas Sembres ${ }^{\star *}$, Mino Randrianarison ${ }^{\star \star \star}$
}

Dans les pays du Sud, les paiements pour services environnementaux (PSE) dédiés à la préservation de la diversité biologique devraient se multiplier à la faveur de la mise en place du mécanisme REDD - dit de « déforestation évitée » et d'abord principalement tourné vers le changement climatique. Les PSE existants rémunèrent plus souvent le coût d'opportunité de I'abandon de certaines activités que la valeur du service fourni, laquelle est difficile à calculer quand il s'agit de biodiversité. Mais cette approche rencontre des problèmes d'efficacité et d'équité. Les PSE peuvent être mis au service du développement et devenir des outils de conservation plus efficaces si l'on dépasse la logique de la compensation du coût d'opportunité.

Mots clés : REDD, paiements pour services environnementaux, biodiversité, déforestation.

Si le carbone a ses « marchés ", la biodiversité a-t-elle les siens ? La biodiversité n'est pas un bien économique, avec ses caractéristiques de dénombrement possible, d'entités isolables et de substituabilité permettant de la rapporter à un équivalent général (Godard, 2005). Ceci rend la possibilité d'une gestion marchande généralisée de la biodiversité assez improbable.

\footnotetext{
* Chercheur au CIRAD, département «Environnements et sociétés », Montpellier, alain.karsenty@cirad.fr

** Économiste du changement climatique, Université de Cambridge, thomas.sembres@cantab.net

*** Doctorante à l'Université d'Antananarivo et à AgroParistech-Engref, mrandria@gmail.com
} 
Il existe bien des échanges marchands autour de la biodiversité, mais rien qui ressemble au marché de type cap-and-trade $e^{1}$ des permis d'émission. Les propositions d'envergure visant à tenter de créer un marché où s'échangeraient des actifs reflétant des niveaux de biodiversité sont rares. On peut mentionner la proposition de Sedjo, Bowes et Wiseman (1991), fondée sur les obligations de conservation, et celles de Panayotou (1994) et de Chomitz (2004) sur les « droits transférables de développement ».

Le système des obligations de conservation impliquerait un "index » des forêts du monde pondérant les valeurs de conservation de chaque type de forêts et le mode de gestion (plantation, forêt secondarisée, exploitation durable, réserve biologique...). Les obligations seraient attribuées en fonction du produit intérieur brut (PIB) par tête (les pays riches auraient d'autant plus d'obligations de conservation à réaliser, soit chez eux, soit à l'extérieur) et de la surface forestière du pays, pondérée par la «valeur de conservation » (ainsi les pays boisés, même pauvres, auraient un minimum d'obligations de protection de leurs forêts). Outre les oppositions politiques prévisibles d'un pays comme le Brésil qui refuse avec constance tout ce qui ressemblerait à une "internationalisation » de la forêt amazonienne, on peut également imaginer les difficultés que poserait l'établissement de l'index pour établir des valeurs de conservation de chaque forêt. Comme le souligne Godard (2005), la diversité des référents collectifs rend la comparaison des éléments de la biodiversité très délicate.

Le système des « droits transférables de développement » (i.e. de déboisement), consiste à distribuer ces droits en quantités limitées aux agents et à leur permettre ensuite de les échanger sur un marché spécifique, à l'image des permis d'émission. Il requiert l'existence d'un seuil minimum légal de superficie devant être maintenu boisé, comme au Brésil où les propriétaires fonciers doivent conserver au moins $80 \%$ de couvert forestier dans l'Amazonie légale. Le mécanisme fonctionne déjà dans des pays développés, fait l'objet d'expérimentation dans certaines régions du Brésil (Chomitz, 2004), et certains analystes pensent possible de le faire fonctionner au niveau mondial (Panayotou, 1994). Mais son application dans les pays du Sud supposerait, d'une part, un programme global d'utilisation des terres reconnu et ratifié par tous les participants et, d'autre part, une généralisation de la propriété privée individuelle ou communautaire alors que la majorité des forêts relèvent du domaine public (White, Martin, 2002).

Ces différents obstacles ont conduit à la proposition sur la « déforestation évitée » formulée en 2005 par un groupe de pays du Sud (Papouasie-NouvelleGuinée, Costa-Rica, etc.) regroupés dans la Rainforest Coalition et placée d'emblée dans le cadre de la négociation pour établir le régime post-Kyoto

1. Qui consiste à fixer une quantité limitée par voie réglementaire, la répartir entre les agents, puis les laisser échanger leurs droits sur un marché ad hoc. 
de lutte contre les changements climatiques. Cette proposition appelée REDD ${ }^{2}$ (Réduction des émissions issues de la déforestation et de la dégradation) depuis la $13^{\mathrm{e}}$ Conférence des parties à la convention-cadre des Nations unies sur les changements climatiques (CCNUCC) en 2007, n'a pas encore fait l'objet d'un accord sur son architecture et ses règles de fonctionnement. L'architecture la plus souvent évoquée consiste à rémunérer des pays qui réduiraient les émissions de $\mathrm{CO}_{2}$ découlant de la déforestation, par rapport à une référence (niveau passé, scénario prédictif ou objectif chiffré convenu) et pour une "période d'engagement " donnée. L'intérêt de la REDD est qu'éviter la déforestation permet aussi de conserver la biodiversité - au contraire du mécanisme de développement propre (MDP) qui ne rémunère que le carbone fixé par les projets de boisement et de reboisement, ne visant ainsi que l'objectif d'atténuation du changement climatique. Mais dans le mécanisme REDD la biodiversité ne constituerait qu'un bénéfice indirect des réductions d'émissions. Si, pour les forêts tropicales denses humides, ces deux objectifs carbone et biodiversité convergeront, les forêts sèches (qui contiennent beaucoup moins de carbone que les premières ${ }^{3}$ mais qui sont riches en diversité biologique) ne bénéficieront sans doute pas des mêmes efforts ou transferts financiers - surtout si c'est un mécanisme de marché qui est retenu.

Un tel mécanisme mondial devra s'appuyer sur des instruments utilisables à l'échelle locale. Comme la création par décret des aires protégées semble atteindre ses limites du fait de contraintes démographiques et sociales dans les pays en développement, on peut considérer que le principe des paiements pour services environnementaux (PSE) occupera une place essentielle. Les PSE ne sont pas nouveaux. En France, le groupe Nestlé a expérimenté la démarche dès la fin des années 1980 pour préserver la source de son eau minérale Vittel des pollutions agrochimiques liées aux pratiques agricoles dans la zone de captage. Le principe était de compenser le coût d'opportunité des agriculteurs correspondant au renoncement aux pratiques susceptibles de polluer la source (plus une somme supplémentaire négociée). Depuis, les PSE se sont multipliés, notamment en Amérique latine. Mais derrière un nom devenu emblématique, ce sont des principes de rémunération très divers qui sont mis en œuvre. La rémunération est généralement calculée sur la base du coût d'opportunité du renoncement aux pratiques existantes et non sur la valeur des services écologiques rendus. C'est le cas de Vittel, qui fait figure de PSE de référence où les paiements ne sont pas conditionnés au changement des taux de nitrate dans la nappe phréatique car

2. Depuis la récente conférence de Copenhague sur le climat en décembre 2009, REDD est devenu « REDD+ " afin d'inclure un ensemble plus large de stratégies carbone dans l'agriculture et la foresterie (pas seulement la réduction de la déforestation et dégradation, mais aussi reforestation, séquestration de carbone dans l'agriculture, etc.).

3. Une forêt soudanaise renferme 7-8 tonnes de $\mathrm{C}$ à I'hectare (biomasse aérienne) tandis qu'une forêt tropicale humide peut en contenir plus de 250 tonnes. 
la contribution de chaque exploitation est impossible à établir (Perrot-Maitre, 2006).

Après un bref rappel sur la définition et les applications des PSE, cet article analyse le rôle important de ces dispositifs dans la mise en œuvre attendue de REDD. La question des coûts, directs et indirects, de l'utilisation des PSE pour réduire la déforestation et la dégradation est ensuite abordée, montrant à quel point l'idée répandue selon laquelle les PSE constitueraient une stratégie REDD «bon marché » est trompeuse. La dernière section de l'article et la conclusion examinent enfin la possibilité d'utiliser les PSE avec plus d'efficacité et d'équité notamment à travers des mesures favorisant l'utilisation des paiements pour l'investissement local.

\section{DÉFINITION ET APPLICATIONS}

Les PSE sont des transactions volontaires et contractuelles entre au moins un acheteur et un vendeur d'un service environnemental bien défini (ou bien d'une pratique agricole ou foncière bien définie) qui débouchent sur un paiement (monétaire ou non) conditionné au respect des termes du contrat sur une période déterminée (Wunder, 2005).

En d'autres termes, les gestionnaires de terres s'engagent sur une durée déterminée à protéger eux-mêmes leur environnement naturel en échange d'une compensation. La nature de la compensation (monétaire ou en nature, individualisée ou groupée, etc.) et les modalités de conservation sont spécifiées dans un contrat. Les expériences de PSE se sont multipliées depuis la fin des années 1980 mais la plupart demeurent des dispositifs extrêmement localisés. Leur taille ne dépasse pas les limites d'un petit bassin versant (les services liés à l'eau sont d'ailleurs au centre de ces dispositifs). Ce n'est que plus récemment que des systèmes de PSE ont été étendus à l'échelle nationale dans quelques pays, peu représentatifs toutefois de l'ensemble des pays tropicaux (Costa Rica, Mexique, Chine). Au Costa Rica, par exemple, le signataire d'un contrat PSE de conservation ${ }^{4}$ reçoit cinq paiements égaux d'environ 64 dollars par hectare et par an, soit 320 dollars en cinq ans, renouvelable (Fondo Nacional de Financiamento Forestal - FONAFIFO, 2006). Entre 1997 et 2006, plus de 6000 contrats ont été signés pour un total dépassant le demi-million d'hectares cumulés.

Alors que les dispositifs de PSE sont restés circonscrits à des contextes particuliers (notamment là où les droits de propriété sont bien définis) et concernent principalement des terres peu productives (c'est-à-dire là où les

4. Le système costaricain prévoit quatre types de contrats PSE : conservation, reboisement, régénération naturelle et systèmes agroforestiers. 
coûts d'opportunité sont abordables), le débat sur la " déforestation évitée " pourrait induire un changement d'échelle de la mise en œuvre des PSE.

\section{LE MÉCANISME INTERNATIONAL DE “ DÉFORESTATION ÉVITÉE 》, CATALYSEUR DES PSE ?}

L'architecture du mécanisme REDD n'est pas encore décidée, et fait l'objet de négociations serrées - qui pourraient se poursuivre jusqu'à la $16^{\mathrm{e}}$ Conférence des parties de la CCNUCC fin 2010. On peut identifier au moins cinq architectures possibles, elles-mêmes susceptibles de variantes :

- 1. Cap-and-trade $e^{5}$ total : un mécanisme qui rémunèrerait les États des pays en développement par des « crédits carbone » correspondant à la réduction constatée des émissions liées à la déforestation (et la dégradation, si la mesure s'avère possible) et qui seraient pleinement utilisables sur le marché mondial du carbone. Il serait mis en place pour une période d'engagement postérieure à 2012, et les "crédits » seraient versés à l'issue de la période d'engagement, sur la base de résultats mesurables. Les réductions seraient constatées au regard d'un scénario de référence (soit une moyenne nationale passée, soit une moyenne régionale ou sous-régionale, soit un scénario futur «business-as-usual») ou d'un objectif chiffré négocié au préalable.

- 2. Cap-and-trade contingenté : la différence concerne la nature des crédits carbone qui seraient délivrés; on peut envisager de verser des "crédits spécifiques REDD » ne pouvant être utilisés que par les pays de l'Annexe $I^{6}$, dans la mesure des engagements supplémentaires de réduction de leurs émissions qu'ils auraient consentis. Ce système de « double marché » revient à contingenter plus ou moins strictement les crédits REDD.

- 3. Mécanisme de marché décentralisé : le mécanisme REDD se calquerait sur les procédures du MDP en élargissant le champ des activités éligibles. Ici, ce sont les projets REDD qui bénéficieraient de crédits carbone certifiés par une tierce partie et non les États (on ne peut pas rémunérer deux fois les mêmes réductions d'émission). Ces crédits pourraient être directement commercialisés sur le marché international des permis d'émission. Notons qu'une telle possibilité avait été discutée lors de la CoP 6 de 2000, mais rejetée du fait notamment des risques inhérents de « fuites » (leakages) : un projet de conservation peut réduire le déboisement là où il est mis en œuvre mais le déplacer sur une autre partie du territoire. C'est d'ailleurs pour cette raison que les propositions initiales relatives au REDD en 2005 étaient construites

5. Système de plafonnement et d'échange (des permis d'émissions de $\mathrm{CO}_{2}$ ).

6. C'est-à-dire les pays qui ont souscrit à des objectifs quantifiés de réduction de leurs émissions ; en règle générale les pays industrialisés du Nord. 
autour d'une architecture « centralisée » (i.e. nationale), même si la possibilité de fuites transfrontalières n'est pas à écarter.

- 4. Fonds international rémunérant les résultats nationaux : dans ce schéma centralisé, les rémunérations vont aux États, mais ne sont pas reliées au marché des permis d'émission, ni négociables sur celui-ci ; un fonds international accorde des rémunérations aux États sur la base des résultats constatés (scénario de référence ou objectif négocié). C'est le principe de la proposition brésilienne de 2005. Dans ce schéma, ce sont les gouvernements récipiendaires qui décident des mesures à prendre et des éventuelles incitations.

- 5. Fonds international pour le financement de politiques et de mesures contre la déforestation : ce schéma s'affranchit de la construction de scénarios nationaux de référence et vise à mobiliser des moyens financiers pour la réalisation de réformes structurelles au niveau national et de programmes de PSE dans les régions menacées par la déforestation, ciblant les acteurs locaux (paysans, communautés, entreprises). Le financement durable d'un tel fonds reste une question ouverte (idem pour le fonds précédent). L'Union européenne envisage qu'une partie de la mise aux enchères des permis d'émission (à partir de 2013) soit affectée à un fonds pour les forêts. Des dispositifs fiscaux, tels que des taxes carbone / énergie internationales par exemple, mais aussi l'idée d'une taxe sur les transactions financières, reprise par la France lors du sommet sur le climat en décembre 2009 à Copenhague, peuvent également être envisagés.

La biodiversité serait sans doute mieux considérée dans une approche " fonds » (options 4 et 5) que dans une approche " marché » dans la mesure où elle ne serait qu'un sous-produit contingent de la vente de crédits carbone alors qu'elle pourrait, dans le cas d'un fonds, être délibérément ciblée comme produit associé. Mais quelle que soit l'option finalement choisie, il est fort probable que des PSE soient utilisés à grande échelle. Avec une architecture basée sur un fonds, les PSE constituent un outil privilégié pour cibler directement les agents de déforestation et leur apporter une aide conditionnée à des changements de pratiques. Avec un mécanisme REDD centralisé de type "marché » (options 1 ou 2), certains États peuvent choisir une stratégie nationale consistant à mettre en place des PSE pour diminuer le taux de déforestation et engranger des crédits carbone si une réduction de la déforestation est effectivement mesurée à l'échelle du pays. Avec un mécanisme de marché décentralisé (option 3), les projets PSE sont en lien direct avec le marché international du carbone - comme ce type de projets possède déjà la possibilité de vendre des réductions d'émissions issues de la « déforestation évitée » sur le marché volontaire de la «compensation carbone $»^{7}$.

7. Sur lequel les projets forestiers représentent environ $1 / 3$ des transactions, tandis que les projets forestiers de boisement / reboisement ne représentent que moins d'un pour mille des projets MDP enregistrés fin 2008. 
Cette articulation entre systèmes de PSE et le mécanisme REDD en construction est d'ailleurs déjà une réalité institutionnelle dans un pays comme le Costa Rica : l'Office costaricain pour la mise en œuvre conjointe permet de négocier la vente de crédits carbone qui aidera à financer le programme national de PSE. Au Brésil, le Fonds Amazonas annoncé par le gouvernement, et auquel la Norvège s'est engagée à verser 1 milliard de dollars d'ici à 2015 (en plus des contributions annoncées de l'État Fédéral brésilien), devrait apporter les financements qui manquent au développement des programmes de paiements compensatoires expérimentés en Amazonas et au Mato Grosso pour les agriculteurs qui réduiraient leur déforestation (AFP, 05/08/2008).

\section{LES PSE SERONT-ILS UNE SOLUTION DE « DÉFORESTATION ÉVITÉE » BON MARCHÉ ?}

\section{L'approche du " rapport Stern "}

Le rapport Stern (2006) - dont la partie sur la réduction de la déforestation a été réalisée par l'Institut international pour l'environnement et le développement $(\text { IIED })^{8}$ (Grieg-Gran, 2006, 2008) - soutient que compenser les acteurs pour la réduction de la déforestation serait une option peu onéreuse dans la gamme des actions possibles pour atténuer les changements climatiques. L'estimation de ces coûts s'est faite à partir de la valeur actualisée d'un certain nombre de productions agricoles impliquées dans la déforestation tropicale. Le coût de la " déforestation évitée » correspondait ainsi à celui des compensations financières à verser sur une base annuelle aux agents responsables de la déforestation. On retrouve bien là l'idée de PSE fondés sur le coût d'opportunité du renoncement à certaines pratiques.

Ce rapport a popularisé l'idée que les PSE visant à réduire la déforestation constituent une option bon marché. Cependant, plusieurs éléments poussent à relativiser une telle conclusion.

\section{Des coûts d'opportunité en hausse}

L'IIED (Grieg-Gran, 2008) a actualisé en mai 2008 son étude sur le coût de la déforestation évitée afin de prendre en compte l'augmentation récente et spectaculaire des prix des produits de base comme l'huile de palme. En deux ans, les estimations de l'IIED ont augmenté de $30 \%$ : le scénario jugé le plus réaliste est passé de 5 à 6,5 milliards de dollars annuels nécessaires pour couvrir les coûts d'opportunité d'une déforestation évitée de 6,2 millions d'hectares annuellement (ce qui revient à diviser le taux de déforestation mondial par

8. International Institute for Environment and Development, basé à Londres. 
deux). De plus, le rapport de l'IIED ne prend pas en compte l'effet rétroactif de la diminution de la déforestation sur les prix agricoles (moins de forêts converties en terres cultivées devrait conduire à une hausse des coûts d'opportunité à travers celle des prix agricoles).

\section{Des coûts de transaction sous-estimés}

Le rapport de l'IIED reconnaît qu'il faut s'attendre à des coûts d'administration substantiels avec les PSE - déforestation évitée, de l'ordre de 4 à 9 dollars annuellement par hectare conservé. Après avoir estimé des coûts de transaction de l'ordre de 6 dollars par an par hectare conservé au Costa Rica (coûts supportés de façon à peu près égale par l'agence nationale chargée de gérer les contrats et les participants au PSE), un pays jouissant d'institutions et de politiques forestières de qualité, ces estimations globales nous semblent sous-estimées.

Le PSE est en réalité un mécanisme qu'il faut construire institutionnellement et cela est coûteux. Les principaux coûts de transaction liés à l'établissement et au suivi des systèmes de PSE tendent à être masqués par l'apparence d'un mécanisme de marché simple et direct.

L'établissement d'un climat de confiance et de coopération est au cœur de la forte institutionnalisation qui s'élabore autour du PSE. Compte tenu de la taille de certains écosystèmes, du dispersement des populations et de la nécessité de mener des actions coordonnées, la mise en place effective de PSE sollicite fortement les institutions locales existantes (conseils de bassin, associations de paysans, associations communautaires d'usagers, syndicats, conseils municipaux) et / ou suscite de nouvelles entités. La visibilité institutionnelle est cruciale pour attirer des financements importants et réduire les coûts de transaction. Wunder et Vargas (2005) indiquent que le manque de confiance et de capital social entre les prestataires et bénéficiaires de services environnementaux est un des obstacles les plus importants à la diffusion des PSE. Et pour de nombreuses populations vivant dans les zones de forêt tropicale, l'État et son entité locale décentralisée sont perçus comme des prédateurs.

Le manque de confiance envers l'État ou des partenaires institutionnels extérieurs peut facilement se cristalliser sur le mécanisme de PSE lui-même. Un PSE mal compris est alors interprété comme un moyen de privatisation des ressources naturelles ou d'exploitation des terres. Parfois, l'aversion au PSE ne tient qu'à la terminologie : le mot « paiement » est une référence directe aux mécanismes de marché et de privatisation si impopulaires dans de nombreux pays en développement ${ }^{9}$. À titre d'exemple, au sein du PSE de Pimampiro dans

9. II est clair que dans de nombreuses régions en développement, les PSE rencontreraient davantage de succès sous le vocable " arrangements solidaires réciproques » qu'avec l'expression idéologiquement marquée de " paiements pour services » ou pire, de « marchés de services environnementaux ». Mais ces références au libre-échange relèvent généralement moins d'une simple 
le nord de l'Equateur, le terme de "paiement » (pago) a été remplacé par celui de "rétribution» (retribución) afin que le mécanisme rencontre davantage de succès auprès des usagers d'eau urbains qui le financent (Wunder, Vargas, 2005). De longs mois sont ainsi parfois nécessaires pour établir une relation de confiance entre les différents acteurs d'un mécanisme de PSE et des remises en question peuvent resurgir périodiquement.

\section{Clarifier les droits de propriété}

Le règlement des conflits sur les droits de propriété est un autre pré-requis institutionnel à l'établissement de PSE. Un système de PSE ne peut fonctionner qu'avec des bénéficiaires bien identifiés et des responsabilités définies. Pour cela, il faut sinon des droits de propriété complets (incluant le droit d'aliéner), au moins des droits d'exclusion reconnus par l'autorité publique et les voisins. Les PSE sont confrontés à un paradoxe de taille : le fonctionnement du mécanisme repose sur l'existence de " maîtrises exclusives ${ }^{10}$, mais son besoin est ressenti d'abord dans les zones où ces droits sont les moins bien définis, là où la constitution de la propriété est un processus souvent conflictuel : fronts pionniers, terres consacrées à l'élevage extensif, etc. En Amazonie brésilienne, par exemple, l'insécurité foncière conduit les acteurs (" propriétaires » ou paysans sans terres « squatters ") à accroître le déboisement au-delà des stricts besoins économiques afin d'affirmer une occupation de l'espace justifiant les prétentions foncières (Araujo et alii, 2008). En Afrique tropicale, les zones forestières relèvent la plupart du temps du régime domanial et les transferts de gestion aux communautés restent d'ampleur limitée (White, Martin, 2002).

La clarification nécessaire des droits d'exclusion est ainsi un défi sérieux et coûteux pour la plupart des projets de PSE. C'est, de plus, un préalable incontournable car, en l'absence de droits clairement définis, les PSE risquent d'exacerber les différends relatifs aux ressources (Mayrand, Paquin, 2004). L'apparente souplesse des PSE, qui, à l'inverse des instruments normatifs tels que la création de zones protégées, chercheraient à changer l'utilisation des sols sans en modifier la propriété, est trompeuse. Même si les PSE n'ont pas l'ambition de redéfinir totalement les droits de propriété à l'instar d'une réforme agraire, leur impact en la matière est loin d'être négligeable : renforcement / clarification des droits pour certains, compétition pour les droits sur les terres réellement contestées et, là où le PSE fait surgir des perspectives de profit, abandon d'un régime de propriété commune (cas du PSE de Pimampiro en Equateur), ou encore remise en cause de contrats locatifs.

maladresse que de la volonté de séduire les bailleurs de fonds occidentaux chez qui les connotations libre-échangistes résonnent positivement.

10. Sur ce terme, voir Le Roy, 1996. 
Enfin, la création d'une "rente de conservation » va susciter des enjeux importants entre les communautés et au sein même de celles-ci. Les conflits au sein de "communautés ", généralement moins homogènes qu'on ne le croit (surtout en Afrique), pour l'appropriation de cette rente de conservation, entraîneront des coûts de prévention et de règlement des conflits (en même temps qu'ils feront apparaître la nécessité d'arbitrages politiques sur la répartition du foncier, et donc de véritables réformes). Mais quand, comme en Côte d'Ivoire, ils prendront une dimension de contestation identitaire de la légitimité des groupes se partageant la terre, les solutions techniques seront impuissantes.

\section{Aléa moral et contrôle de l'additionnalité}

Une fois en place, les PSE doivent faire face à deux problèmes sérieux qui suscitent de nouvelles dépenses : les problèmes liés à l'additionnalité environnementale du mécanisme et les risques d'aléa moral («passager clandestin »).

Le PSE est un dispositif conditionnel, c'est-à-dire que les paiements sont assujettis à la délivrance de services environnementaux effectifs, services qui n'auraient pas été délivrés sans les paiements. Mais le suivi de l'additionnalité environnementale d'un dispositif est complexe. D. Kaimowitz (2008) souligne le fait que les programmes de PSE de conservation des forêts au Costa Rica sont critiquables sur ce point :

«Les résultats des évaluations économétriques de l'impact du programme de PSE au Costa Rica sur la réduction de la déforestation sont mitigés. Certains suggèrent que le programme n'a que modestement réduit la déforestation, et d'autres que son impact en ce sens a été négligeable (...). Toutes les études reconnaissent que de nombreux propriétaires terriens ayant reçu des paiements auraient conservé leur forêt même en l'absence de paiements et que le ralentissement de la déforestation au Costa Rica n'est pas dû principalement à la mise en place du PSE. ${ }^{11}$

Les fortes asymétries d'information entre les intermédiaires institutionnels chargés de conclure les contrats (ONG, ingénieurs forestiers, etc.) et les participants impliquent des risques importants d'aléa moral. Le risque d'opportunisme avant et pendant un contrat de PSE est élevé, notamment pour les programmes qui concentrent les paiements en début de période (cas des contrats de reforestation des PSE costaricains avec $46 \%$ des versements effectués la première année et $6 \%$ les neuf années suivantes).

Par ailleurs, les PSE induisent une redistribution de ressources, souvent pour une part très importante du revenu des personnes concernées. Cette redistribution risque d'accentuer les inégalités sociales et les situations de pauvreté relative.

11. "Econometric studies that have assessed how much Costa Rica's PES program reduced deforestation have yielded mixed results. Some suggest the program has achieved modest reductions, others that the effect has been negligible (...). The studies all agree that many landowners who received payments would have conserved their forest even without them and that the decline in Costa Rica's national deforestation rates cannot be attributed principally to the payments » (p. 489). 
D. Kaimowitz (2008) souligne les compromis de tels programmes entre les objectifs d'efficacité et les considérations d'équité ( « Le programme de PSE au Costa Rica a essentiellement bénéficié à des sociétés et des grands propriétaires ${ }^{12}$ ).

En dépit de sa notoriété, le système de PSE au Costa Rica n'est pas un exemple de mécanisme qui prend sérieusement en compte les considérations sociales puisque les petits exploitants et les populations pauvres sont largement exclus du système (Zbinden, Lee, 2005). Il est vrai qu'il n'a pas été conçu dans l'objectif de réduire la pauvreté. Une partie substantielle des coûts de transaction liés à l'établissement des contrats de PSE (plan d'aménagement avec un ingénieur forestier...) sont laissés à la charge des participants. Le FONAFIFO aurait pu se charger de ces coûts afin de faciliter la participation des petits propriétaires terriens, mais ce choix n'a pas été fait.

S’ils doivent devenir des instruments utilisés à grande échelle, on voit mal comment les systèmes de PSE pourraient éviter de présenter des buts sociaux et ne pas être aménagés pour tenir compte de la participation des populations les plus pauvres et de l'impact sur l'emploi ou sur la sécurité alimentaire. Cette prise en compte des impacts sociaux se traduit une nouvelle fois en surcoûts et / ou en perte d'efficacité environnementale (additionnalité). Par exemple, les rapports d'évaluation du $\mathrm{SLCP}^{13}$ (système chinois qui se rapproche le plus d'un mécanisme de PSE) montrent que les taux de survie des plantations sont très souvent en-deçà de ceux stipulés dans les contrats, mais qu'il est néanmoins impossible d'annuler les paiements puisque ce serait abandonner les zones où la pauvreté est la plus sévère (Bennett, Xu, 2005). Conditionnalité et additionnalité des PSE sont donc souvent atténuées afin de ne pas exclure les plus pauvres.

\section{D'inéluctables effets de " contagion "}

Lorsque l'IIED estime qu'environ 6,5 milliards de dollars sont nécessaires annuellement pour réduire de moitié la déforestation mondiale, c'est en faisant l'hypothèse qu'il sera possible de concentrer les paiements sur les seules forêts menacées. Cette hypothèse ne prend pas en compte la possibilité que les acteurs résidant dans les zones non concernées par les paiements fassent pression pour bénéficier eux aussi de ces transferts, au nom de l'équité et de la récompense due aux pratiques « spontanées » de conservation ${ }^{14}$. Ce type d'argument est facilement mis en avant par les communautés, notamment au sein des populations " autochtones ». Il a son pendant au plan international, avec l'exemple des pays d'Afrique centrale qui demandent, dans le cadre des

\footnotetext{
12. "Most benefits from Costa Rica's PES program have gone to companies and large individual landowners » (p. 489).

13. Sloping Land Conservation Programme (SLCP).

14. Pratiques spontanées de conservation qui peuvent être bien souvent simplement l'absence d'opportunité de conversion de la forêt à d'autres usages (auquel cas il n'y aurait pas d'additionnalité d'un paiement pour la conservation).
} 
négociations REDD, des rétributions financières pour avoir préservé l'essentiel de leurs massifs forestiers originels.

L'effet de contagion reste très difficile à quantifier, mais il fait peu de doute que les PSE s'encombrent de zones peu ou pas menacées de déforestation pour s'assurer la protection, sans fuites, d'une petite proportion de forêts réellement menacées. Muñoz et alii (2006) estiment que seulement entre $11 \%$ et $28 \%$ des hectares inclus dans le programme de PSE mexicain en 2003 et 2004 présentaient un risque élevé ou très élevé de déforestation. Avec la perspective d'une généralisation des PSE dans les pays tropicaux, on voit mal comment, politiquement, il serait possible de concentrer les paiements sur les seules forêts menacées.

\section{LES PSE PEUVENT-ILS ÊTRE MIS AU SERVICE DU DÉVELOPPEMENT ?}

\section{Des rémunérations non indexées sur la valeur du " service "}

En théorie, les PSE doivent correspondre à la valeur des services fournis. En pratique, cela est rarement possible. Pour la biodiversité, l'absence de marché et la variabilité du contenu exact que recouvre le concept de biodiversité rendent improbable le recours à des " prix ». Ces obstacles ont conduit une organisation comme Conservation International $(\mathrm{CI})$ à rémunérer les acteurs locaux pour le coût d'opportunité du renoncement à une activité jugée dangereuse pour l'environnement, comme l'exploitation forestière, certaines pratiques de pêche ou le déboisement.

Le rapport Stern (2006), estime que le coût d'opportunité à compenser aux ménages agricoles pour parvenir à réduire de $50 \%$ la déforestation est de 300 dollars par an, soit moins d'un dollar par jour. Si l'analyse devait s'arrêter là, des questions d'équité pourraient légitimement être posées. Quand des populations gagnent l'équivalent d'un ou deux dollars par jour (soit la limite du seuil de la grande pauvreté) en dégradant leur environnement, les compenser à hauteur du coût d'opportunité de l'arrêt de leur activité ne répond pas aux aspirations fondamentales des individus à sortir de la misère et accéder à une vie décente.

\section{De la compensation du coût d'opportunité à l'investissement dans de nouvelles pratiques agraires}

Un autre problème est lié à l'efficacité potentielle de tels paiements. Sans changement en profondeur des modes d'utilisation du milieu, qui passe notamment par une évolution des pratiques agricoles, la compensation pour le coût d'opportunité risque de ne rien changer à la situation, notamment si les revenus compensés sont peu élevés et ne permettent pas d'acquérir le capital 
nécessaire à la mise en œuvre de nouveaux itinéraires techniques de production agricole ou agroforestière. Il est en outre difficile de prévoir ce que les agents feront de l'argent qui leur sera distribué : la consommation immédiate peut être préférée à l'épargne et à l'investissement, avec la tentation de reprendre les pratiques abandonnées par contrat une fois les revenus consommés. C'est une logique de versement sans fin ${ }^{15}$ d'une rente qui se dégage de ce type de paiements, même s'il s'agit bien souvent de " rentes de pauvres ».

Il est cependant possible d'envisager un rôle pour les PSE, au-delà du principe de compensation du coût d'opportunité. Deux principes peuvent être proposés :

- ne pas se contenter de compenser pour les coûts d'opportunité, mais y ajouter une subvention d'investissement ad hoc limitée dans le temps pour les coûts de l'aménagement de zones de culture permanentes et l'adoption de nouvelles techniques agricoles durables. Cela suppose parallèlement une offre systématique d'alternatives en termes d'itinéraires techniques agricoles, de crédit rural et de procédures de sécurisation foncière par l'enregistrement et la cartographie des droits locaux ;

- utiliser une monnaie spécifique plutôt que de l'argent pour les paiements. Une telle monnaie serait dédiée à l'acquisition des moyens nécessaires (intrants, journées de travail, relevés cadastraux...) pour faire évoluer les pratiques agricoles et construire une alternative durable à l'abattis-brûlis susceptible d'accroître les revenus locaux.

Le principe de la subvention n'a de sens qu'inséré dans un dispositif proposant des alternatives viables aux ruraux. Cela passe par de l'outillage, de la formation, un programme visant à sécuriser le foncier, du crédit et de l'assurance... Tout cela sera forcément onéreux et rendra sans doute moins attractive financièrement l'option de la lutte contre la déforestation à travers des PSE. Mais c'est la seule manière d'envisager une réelle efficacité à terme. La durée de ces subventions pourrait être limitée dans le temps, de 5 à 10 ans selon les besoins et les contextes.

Quant à la monnaie spécifique, elle permet de limiter les achats aux usages fixés par le contrat : remplacement de denrées et biens, achat de matériel et de services agricoles... Elle permet également de stimuler l'économie locale des biens et services à travers les politiques d'achat de la structure commerciale nécessaire pour assurer l'interface entre les groupes ciblés par le mécanisme et les fournisseurs. Un dispositif institutionnel ad hoc est indispensable pour assurer l'échange de la monnaie spécifique en argent, dans les deux sens. Le schéma ci-dessous a été proposé à Madagascar (Karsenty, 2008) dans le cadre de la préparation d'un projet de gestion de la biodiversité dans la forêt d'Ambohilero (commune de Didy).

15. À l'inverse, les projets MDP dans le domaine industriel génèrent des crédits sur une période de temps limitée, allant de 7 à 21 ans, et visent à des changements de trajectoire à travers l'adoption de nouvelles technologies. 
Figure 1 : Schéma d'un PSE pour la réduction du déboisement agricole annuel faisant appel à une monnaie spécifique pour orienter les dépenses vers l'investissement local

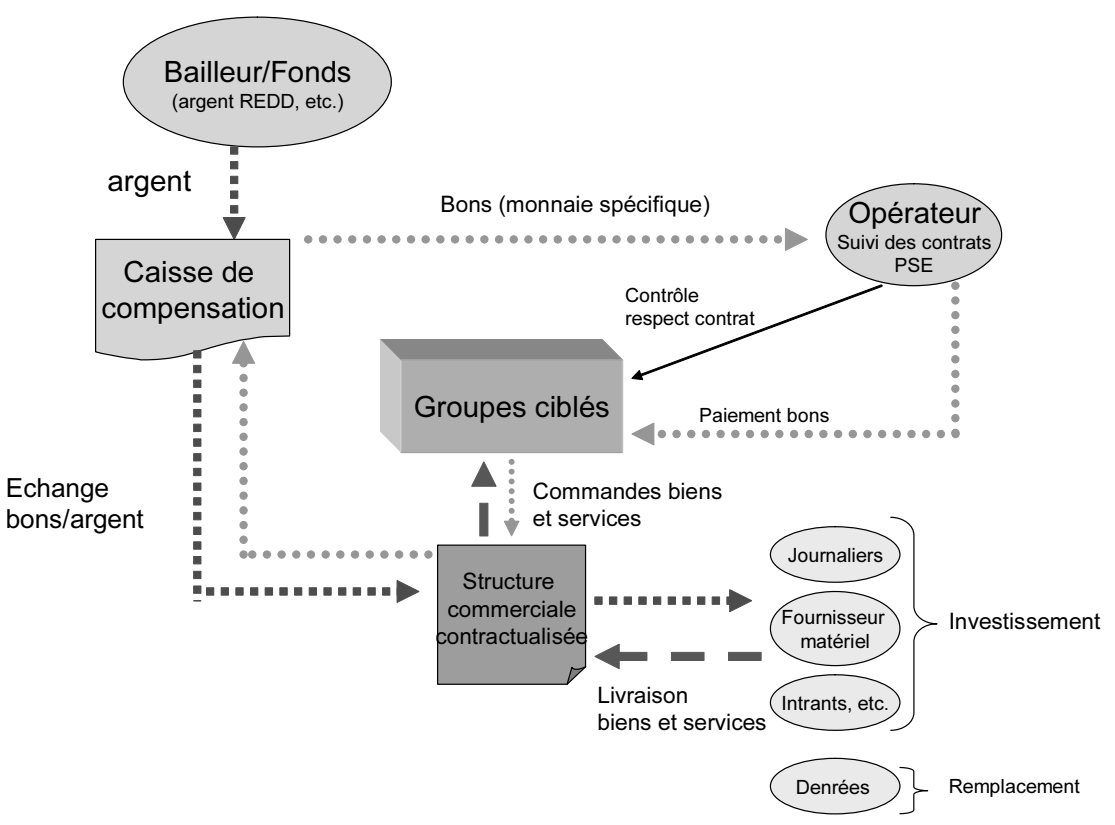

Dans ce schéma, une caisse de compensation assure la conversion des deux types de monnaie et une structure commerciale assure l'interface entre les ménages (occupants des terres) et les fournisseurs (de matériel et d'expertise pour changer les pratiques agricoles). La monnaie spécifique (sous la forme de bons d'achats) est initialement confiée à l'opérateur chargé de la mise en œuvre et du suivi des PSE. Les bons sont ensuite distribués aux ménages en fonction du respect du contrat. Les ménages ou groupes qui détiennent ces bons s'adressent enfin à une structure locale (coopérative communale ou commerçant participant au programme) qui fournit directement le matériel et l'expertise demandée.

Encore faut-il que les ménages ruraux soient en mesure de maîtriser les dimensions techniques et économiques de nouvelles pratiques agricoles. Réduire le risque d'échec supposerait qu'un tel dispositif soit accompagné d'un programme intégré d'appui et de formation agricole, afin de pouvoir accompagner les paysans dans les changements d'itinéraires techniques nécessaires. On voit qu'on s'éloigne ainsi de plus en plus de l'instrument « bon marché » évoqué implicitement dans le rapport Stern, pour se rapprocher du concept mis en œuvre par Vittel où les paiements ont été calibrés en fonction du coût de l'adoption de nouvelles pratiques agricoles. Ce principe d'un paiement dessine toutefois ce 
qui pourrait être une ligne de partage entre des PSE rattachés à une logique de paiement de « rentes » (même modestes) et des PSE tournés vers le financement d'un développement local ménageant les ressources environnementales efficace et autonome sur le long terme.

Une telle orientation des PSE n'est pas généralisable à toutes les situations. Ce schéma est inopérant pour changer la décision de l'agro-businessman qui s'apprête à déboiser une forêt dégradée pour établir une plantation extensive de soja ou de palmier à huile, deux causes immédiates de déboisement massif à l'échelle mondiale. Là, le recours à la loi (quand elle protège les forêts) ou à d'autres modes de distribution du foncier semblent être plus appropriés.

\section{CONCLUSION}

Une certaine classe de paiements pour services environnementaux, ceux qui visent à protéger les forêts dans les pays du Sud, pourrait connaître une expansion inédite dans ces pays avec la mise en œuvre, possible, du mécanisme REDD ou REDD+ après 2012. Bien que tourné en priorité vers l'objectif de réduction des émissions de gaz à effet de serre, ce dernier doit sa popularité au fait qu'il permet de combiner des objectifs propres aux deux grandes conventions internationales sur l'environnement, celle sur le climat et celle sur la diversité biologique. La biodiversité présente dans les forêts tropicales constituerait ainsi un bénéfice dérivé de la lutte contre les changements climatiques au niveau des forêts, ce qui pourrait lui permettre d'avoir des financements aujourd'hui impossibles à mobiliser à grande échelle pour cela.

Si la nature des services environnementaux que l'on rémunère n'est pas ambiguë dans les contrats (du fait de l'usage d'indicateurs ad hoc), la rémunération elle-même mérite discussion. Dès que de la biodiversité est concernée (ce qui n'est pas le cas des MDP forestiers, non traités dans cet article), les paiements ne sont pas, en règle générale, proportionnés à la valeur du « service environnemental rendu »- valeur bien souvent difficile à calculer - mais plutôt à celle du coût d'opportunité entraîné par le renoncement à certaines pratiques. Sur une telle base de calcul, les PSE peuvent sembler constituer un instrument permettant de préserver des forêts tropicales à des coûts modérés. Mais ce serait sous-estimer les coûts, y compris politiques, liés à une clarification des droits de propriété susceptibles d'entraîner une plus grande sécurité foncière. En outre, les coûts de transaction attachés à la mise en place d'un grand nombre de contrats locaux et à leur suivi pourraient s'avérer beaucoup plus élevés que prévu : il s'agit d'effectuer des constructions institutionnelles nouvelles, de bâtir la confiance et de se prémunir contre les risques d'aléa moral. Enfin, si l'idée de se concentrer sur les seules forêts menacées à court terme de déforestation permet d'envisager de limiter le nombre des contrats de PSE, on ne peut exclure l'apparition d'un 
" effet de contagion " provoqué par les revendications des groupes et individus résidant dans des espaces boisés mais en dehors des zones de programmes PSE.

La principale critique apportée aux programmes de PSE est que les objectifs de réduction de la pauvreté sont secondaires, voire tout simplement absents. Quand les coûts d'opportunité sont bas du fait de la forte pauvreté, et que les rémunérations envisagées pour ce type de programmes ne dépassent pas 1 dollar par jour et par ménage, on peut s'interroger sur l'équité de contrats qui réduisent objectivement les possibilités des ruraux de sortir de la pauvreté en convertissant l'espace forestier en terres agricoles. Certes, la plupart des formules de PSE proposées par les organisations de conservation envisagent qu'en sus du coût d'opportunité, des actions de développement communautaires soient entreprises. Mais visiblement de telles dimensions restent secondaires par rapport à l'objectif de conservation rémunérée, et elles ne sont pas pensées dans une perspective globale de transformation des modes d'utilisation du milieu, perspective qui nécessite des actions coordonnées à plusieurs échelles et soutenues dans le temps. Sur ce point, on peut dessiner une conception quelque peu différente des PSE, moins orientée vers le versement sans fin d'une rente de conservation mais plus tournée vers la constitution d'un vecteur de transfert de capitaux nécessaires au changement d'itinéraires techniques dans les systèmes de production locaux. L'utilisation d'une monnaie spécifique pour orienter les dépenses des bénéficiaires des transferts vers des investissements compatibles avec les objectifs de conservation de la biodiversité constituerait un instrument potentiellement intéressant pour accompagner la transition proposée.

Avec l'intérêt croissant pour les instruments économiques dans le champ de l'environnement, les PSE sont en passe de devenir dans les discours des organisations internationales la nouvelle panacée pour atteindre les grands objectifs des conventions sur l'environnement. À l'évidence ils ne le sont pas, d'autant qu'une grande partie des activités menaçantes pour la biodiversité est manifestement hors de portée de ce type de paiements, du fait des coûts d'opportunité très élevés que représenterait leur abandon. L'augmentation probable de ces coûts d'opportunité à l'avenir, à travers la demande accrue de terres mobilisables pour l'agriculture, constitue d'ailleurs l'une des limites les plus manifestes de leur déploiement dans le temps et l'espace. Ils semblent pourtant devoir s'imposer comme un instrument incontournable, complétant les instruments réglementaires et légaux qui restent indispensables. On peut penser que leur efficacité dépendra non seulement des fonds mobilisables pour mener des programmes à grande échelle, mais aussi et peut-être surtout de leur insertion dans des politiques publiques transsectorielles, agissant simultanément sur un ensemble de facteurs clés, comme le foncier, l'agriculture et l'emploi rural. L'efficacité des PSE passe par leur intégration dans des politiques publiques 


\section{d'environnement qui abordent les dynamiques rurales dans leurs différentes dimensions.}

\section{BIBLIOGRAPHIE}

Araujo C., Araujo Bonjean C., Combes J.-L., Combes Motel P., Reis E. J., 2008, Land Reform and Deforestation in the Brazilian Amazon, Présenté à l'atelier international du CERDI sur la déforestation, 14 novembre 2008, Clermont-Ferrand.

Bennett M. T., Xu J., 2005, China's Sloping Land Conversion Program: Institutional Innovation or business as Usual ?, ZEF-CIFOR Workshop on «PSE - Methods and Design in Developed and Developing Countries », Titisee, 1618/06/2005.

Chomitz K., 2004, "Transferable Development Rights and Forest Protection: An Exploratory Analysis ». International Regional Science Review, 27, pp. 348-373. [online] URL : http: //irx.sagepub.com/cgi/reprint/27/3/348

FONAFIF0, 2006, "Distribución del Pago de los Servicios Ambientales por Modalidad, Periodo 1997-2007 », www.fonafifo.com

Godard 0., 2005, Les conditions d'une gestion économique de la biodiversité - Un parallèle avec le changement climatique, Cahier $n^{\circ}$ 2005-018 du Laboratoire d'économétrie de l'École polytechnique / CNRS.

Grieg-Gran M., 2006, The Cost of Avoiding Deforestation - Report prepared for the Stern Review of the Economics of Climate Change, IIED.

Grieg-Gran M., 2008, The Cost of Avoiding Deforestation. Update of the Report prepared for the Stern review of the Economics of Climate Change, IIED.

Kaimowitz D., 2008, " The Prospects for Reduced Emissions from Deforestation and Degradation (REDD) » in « Mesoamerica » International Forestry Review 10 (3), pp. 485-495.
Karsenty A. 2008, Chaîne de valeur du bois d'œuvre et perspectives de mise en place de paiements pour services environnementaux dans la forêt d'Ambohilero (Madagascar), Rapport de mission, Antananarivo, Projet Gesforcom (Union européenne) et Cirad.

Le Roy E., 1996, "La théorie des maîtrises foncières " in Le Roy E., Karsenty A., Bertrand A., La sécurisation foncière en Afrique, pour une gestion viable des ressources renouvelables, Paris, Karthala.

Mayrand K., Paquin M., 2004 Le paiement pour les services environnementaux : Étude et évaIuation des systèmes actuels, Montréal, Commission de coopération environnementale de l'Amérique du Nord.

Muñoz C., Guevara A., Bulás J. M., Torres J. M., Braña J., 2006, "Pagar por los servicios hidrológicos del bosque en México » in Pagiola S., Bishop J., Landell-Mills N. (dir.), La venta de servicios ambientales forestales, $2^{\mathrm{e}}$ édition, Mexico, INE.

Panayotou T., 1994, "Conservation of Biodiversity and Economic Development: The Concept of Transferable Development Rights » Environmental and Resource Economics, 4, pp. 91110.

Perrot-Maitre D., 2006, « The Vittel Payment for Ecosystem Services: a "Perfect »PES Case? ", International Institute for Environment and Development, Londres, http://www. iied.org/NR/forestry/projects/water.html

Sedjo R. A., Bowes M., Wiseman C., 1991, "Toward a Worldwide System of Tradeable Forest Protection and Management Obligations ", RFF Discussion Paper ENR91-16, Resources for the Future, Washington D. C. 
Alain Karsenty, Thomas Sembres, Mino Randrianarison

Stern N., 2006, « Stern Review on the Economics of Climate Change ", http://www.hm-treasury. gov.uk/independent_reviews/stern_review_ economics_climate_change/stern_review_ report.cfm

White A., Martin A., 2002, "Who Owns the World's Forests? Forest Tenure and Public Forests in Transition », Forest Trends and Center for International Environmental Law, Washington D. C.
Wunder S., 2005, «Payments for Environmental Services: Some Nuts and Bolts », CIFOR Occasional Paper, 42, $26 \mathrm{p}$.

Wunder S., Vargas M. T., 2005, Beyond "Markets », Why Terminology Matters. http://www. naturabolivia.org/Informacion/-Beyond\%20ma rkets.pdf [consulté 07/2007].

Zbinden S., Lee D. R., 2005, « Paying for Environmental Services: an Analysis of Participation in Costa Rica's PSA Program », World Development, vol. 33, $n^{\circ}$ 2, pp. 255-272. 